\title{
Estudio de la deformación miocárdica longitudinal en pacientes con cardiopatía isquémica
}

\section{Study of longitudinal myocardial deformation in patients with ischemic cardiopathy}

Aylen Pérez-Barreda', Idalys Román-Fernández²*, Amalia Peix-González', Ángel Y. Rodríguez-Navarro², Óscar A. Alfonso-Montero y Adrián Naranjo-Domínguez ${ }^{1}$

${ }^{1}$ Instituto Cubano de Cardiología y Cirugía Cardiovascular; ${ }^{2}$ Centro de Investigaciones Médico Quirúrgicas, Unidad de Imagen Cardiovascular. La Habana, Cuba

\begin{abstract}
Resumen
Introducción: Las enfermedades cardiovasculares constituyen la primera causa de muerte en Cuba y la mayoría de los países desarrollados. La ecocardiografía con speckle tracking bidimensional (ST 2D) es una técnica reciente en la evaluación de la función cardíaca. Objetivos: Determinar la relación entre la deformación miocárdica medida por ST 2D y el estado de la circulación coronaria en pacientes con cardiopatía isquémica, en el CIMEQ, durante un año. Material y método: Se realizó un estudio analítico y transversal con 55 pacientes con indicación de coronariografía sometidos a ecocardiograma bidimensional y estudio de ST 2D con medición de la deformación longitudinal (DLG). Se crearon dos grupos: enfermedad coronaria significativa $(E C S=32)$ y no significativa $(E C N S=23)$. Se utilizó SSPS para análisis de los resultados. Resultados: La edad promedio fue mayor en la ECS (55.6 \pm 9.3 vs. $61.8 \pm 8.8 ; p=0.014)$. Predominaron los hombres con ECS $(47.3 \%)$, los hipertensos ( $E C S=90.6 \%$ y ENCS $=65.2 \% ; p=0.02$ ) y los fumadores $(E C S=59.4 \%$ y ENCS $=17.4 \% ; p=0.002)$. El diagnóstico más frecuente fue la angina crónica estable (87\%). En la ECS predominó la enfermedad de tres vasos (75\%). La DLG fue menor en la ECS [(-20.0 \pm 3.2 vs. $-22.1 \pm 3.6 ; p=0.035)$; AUC $=0.458]$. No hubo diferencias en la DLG según el número de vasos significativamente afectados. Conclusiones: Los resultados encontrados no justifican el empleo del ST 2D para diferenciar la ECS.
\end{abstract}

Palabras clave: Cardiopatía isquémica. Ecocardiografía con speckle tracking 2D. Deformación miocárdica longitudinal. Función cardíaca.

\begin{abstract}
Introduction: Cardiovascular diseases are the leading cause of death in Cuba and most of the developed countries. Two-dimensional speckle tracking echocardiography (2D ST) is a recent technique in the evaluation of cardiac function. Objectives: To determine the relationship between myocardial deformation measured by $2 D S T$ and coronary circulation in patients with ischemic heart disease, in the CIMEQ, for 1 year. Material and method: An analytical, cross-sectional study was carried out with 55 patients with an indication for coronary angiography who underwent $2 D$ echocardiography and $2 D$
\end{abstract}

\section{Correspondencia:}

*Idalys Román-Fernández

E-mail: idalys8825@gmail.com
Fecha de recepción: 14-07-2019

Fecha de aceptación: 06-02-2020

DOI: 10.24875/ACM.20000290
Disponible en internet: 09-03-2020 Arch Cardiol Mex. 2020;90(3):300-308 www. archivoscardiologia.com BY-NC-ND (http://creativecommons.org/licenses/by-nc-nd/4.0/). 
ST study with longitudinal strain measurement (LSM). Two groups significant coronary disease $(S C D=32)$ and not significant $(N S C D=23)$ were created. SSPS was used to analyze the results. Results: The average age was higher in SCD (55.6 \pm 9.3 vs. $61.8 \pm 8.8, p=0.014)$. Men with $S C D(47.3 \%)$, hypertensive $(S C D=90.6 \%$ and $N S C D=65.2 \%, p=0.02)$ and smokers $(S C D=59.4 \%$ and $N S C D=17.4 \%, p=0.002)$ predominated. The most frequent diagnosis was chronic stable angina (87\%). Three-vessel disease (75\%) prevailed in SCD. The LMS was lower in SCD ([-20.0 \pm 3.2 vs. $-22.1 \pm 3.6, p=0.035]$; $A U C=0.458)$. There were no differences in $L S M$ according to the number of significantly diseased vessels. Conclusions: The results found do not justify the use of $2 D S T$ to discriminate $S C D$.

Key words: Ischemic cardiopathy. 2D speckle tacking echocardiography. Longitudinal myocardial deformation. Cardiac function.

\section{Introducción}

Las enfermedades cardiovasculares constituyen la primera causa de muerte en la mayoría de los países desarrollados ${ }^{1}$.En la actualidad, sólo por esta causa fallecen cada año más de 20000 cubanos $^{2}$.

La cardiopatía isquémica es una enfermedad en la que se comprometen la perfusión miocárdica y la oxigenación tisular, lo cual ocasiona modificaciones en la función ventricular ${ }^{3}$.

Evaluar el efecto de la cardiopatía isquémica sobre la función cardíaca es uno de los objetivos sistemáticos de los laboratorios de ecocardiografía ${ }^{4}$. La variable ecocardiográfica más utilizada para cuantificar la función sistólica del ventrículo izquierdo (VI) es la fracción de expulsión del VI (FEVI) y su determinación mediante ecocardiografía depende, de manera crucial, del conocimiento experto del operador, además de que se modifica por una variabilidad intraobservador e interobservador significativa ${ }^{5}$.

La FEVI proporciona un índice general de la función de la cámara del VI, sin tener en cuenta el papel relativo de los diferentes componentes de la función miocárdica (deformación en diversas direcciones y rotación), que pueden afectarse en grados diversos, a pesar de que la FEVI continúe en valores normales ${ }^{6,7}$.

La ecocardiografía con análisis de la deformación (strain; STE 2D) miocárdica se ha convertido en una técnica regular para la valoración de distintas cardiopatías. El estudio mediante rastreo de marcas ha emergido como una novedosa técnica para la evaluación cuantitativa de la función global y segmentaria del miocardio.Las alteraciones de la deformación miocárdica suelen presentarse antes de las alteraciones visuales en el movimiento regional de la pared miocárdica, lo cual le confiere valor a su estudio ${ }^{8,9}$.

Las técnicas como el speckle tracking (STE) han mejorado la evaluación no invasiva de la deformación miocárdica respecto de la bidimensional convencional y aportan una información exacta en las fases iniciales de las enfermedades del miocardio ${ }^{10}$.Los datos de la deformación se obtienen mediante una medición automática, fotograma a fotograma, de la distancia entre dos puntos de cada segmento del VI durante el ciclo cardíaco en tres dimensiones (radial, circunferencial y longitudinal) ${ }^{11,12}$.

La coronariografía ha sido la técnica diagnóstica invasiva por excelencia en el estudio anatómico de la enfermedad arterial coronaria. Aunque por lo general ésta es una investigación segura, no está exenta de complicaciones, además de que su costo es eleva$\mathrm{do}^{13,14}$. Por lo tanto, se requieren métodos simples, no invasivos, que mejoren la selección y pronósticos de los pacientes remitidos para coronariografía 4 . A pesar de los numerosos trabajos que acreditan la utilidad de la técnica en condiciones de reposo, recientes publicaciones la ponen en duda en ese contexto ${ }^{15,16}$; esto ha llevado a realizar este trabajo con el objetivo de determinar la relación entre la deformación miocárdica medida por speckle tracking 2D y el estado de la circulación coronaria en pacientes con sospecha de cardiopatía isquémica.

\section{Método}

Se condujo un estudio observacional, analítico y transversal durante los meses de enero de 2016 a enero de 2017 en el hospital CIMEQ. La muestra se integró con todos los pacientes que acudieron al laboratorio de hemodinámica para someterse a coronariografía con el diagnóstico clínico de cardiopatía isquémica. La muestra quedó conformada por 55 pacientes con base en los siguientes criterios: mayores de 18 años, pacientes con ventana acústica óptima e individuos firmantes del consentimiento informado, con exclusión de los pacientes con enfermedades oncológicas, valvulopatías graves, alteraciones de la contractilidad en reposo y trastornos del ritmo cardíaco.

Se estudiaron las siguientes variables: edad, sexo, factores de riesgo aterogénico, presencia de enfermedad coronaria, extensión y arteria afectada, así como 
las relacionadas con el ecocardiograma en modo $\mathrm{M}$, bidimensional y deformación miocárdica, cuyos valores normales se tomaron en cuenta según las recomendaciones para la cuantificación de las cámaras cardíacas en adultos de la American Heart Association y la European Association of Cardiovascular Imaging ${ }^{17}$.

Se examinó a los participantes en el estudio mediante ecocardiografía transtorácica mediante el ecógrafo comercial EPIQ7 ultrasound system (Philips Medical Systems) equipado con un transductor de $2.5 \mathrm{MHz}$. Todas las imágenes se obtuvieron con la misma frecuencia de fotogramas (50-80 cuadros/s). Se determinó el momento de cierre de la válvula aórtica al examinar el movimiento de dicha válvula en la proyección apical de eje largo.

El análisis de las imágenes de speckle tracking se efectuó con el programa informático comercial QLab. Se utilizaron las tres proyecciones apicales $4 C, 2 C$ y $3 \mathrm{C}$ para las determinaciones de la deformación longitudinal segmentaria y global. El programa marcó de manera automática los bordes endocárdico y epicárdico, además de dividir de modo automático cada proyección ecocardiográfica y considerar la segmentación en 17 segmentos que guarda relación con el área de perfusión de las arterias epicárdicas. Esto proporcionó una confirmación automatizada del tracking, expresada en porcentajes segmentario y global. Los valores de la deformación miocárdica se presentan en forma de diagrama de ojo de buey (mapa polar).

\section{Protocolo de coronariografía}

Para la coronariografía diagnóstica se emplearon las técnicas comunes de este procedimiento. Se utilizó el equipo Integris HM 3000-Philips Medical Systems-Nederland B. V. Se usó el programa de cuantificación Phillips para la línea Integris en la angiografía coronaria cuantitativa.

\section{Análisis estadístico}

El análisis estadístico se realizó con ayuda del paquete Statistical Package Scientific System SPSS. Se usó la prueba de Kolmogorov-Smirnov para evaluar la normalidad de las variables. Se emplearon medidas de resumen para datos cualitativos (razones, porcentajes) y medidas de resumen para datos cuantitativos (media aritmética, desviación estándar). Se utilizó $\chi^{2}$ para comparación de grupos con variables cualitativas. La t de Student se usó para comparar variables continuas (de acuerdo con las características de su distribución) entre dos grupos y el Anova de un factor para la comparación entre más de dos grupos. Se tomó como punto de significación 0.05 .

\section{Aspectos éticos}

En la investigación se respetaron las bases éticas de las exploraciones en seres humanos. Se obtuvo la aceptación libre del paciente, previamente informado, de que se sometería a procedimientos diagnósticos inocuos.

\section{Limitaciones}

Entre las limitaciones de la investigación es preciso mencionar que la muestra utilizada es pequeña, ya que se conformó con los pacientes sometidos a coronariografía en el laboratorio de hemodinámica del CIMEQ, sitio que recibe la menor cantidad de urgencias en Cuba.

\section{Resultados}

Se estudió a 55 pacientes, 38 (69.1\%) de ellos del sexo masculino. La edad promedio fue de 59 años. Hubo $32(58.2 \%)$ casos con ECS. Casi la mitad de los enfermos correspondió a hombres con ECS (47.3\%). La edad promedio fue significativamente mayor en los pacientes con ECS $(55.6 \pm 9.3$ vs. $61.8 \pm 8.8 ; p=0.014)$.

Los factores de riesgo cardiovascular evaluados fueron todos más frecuentes en el grupo de los sujetos con ECS. El factor de riesgo que se presentó más a menudo, tanto en individuos con ECS como en los que tenían ECNS, fue la HTA, aunque fue más común entre los primeros $(90.6 \%)$. Llama la atención que casi el $60 \%$ de los que presentaron ECS era fumador, con una diferencia notoria entre los dos grupos $(p=0.002)$.

En relación con el diagnóstico clínico, fueron más frecuentes los casos con angina crónica estable (48 pacientes, $87.2 \%$ ), que representaron más de las tres cuartas partes de todos los pacientes.

De los individuos con ECS, se observó un predominio de los que tenían enfermedad arterial de tres vasos, presente en el $75 \%$ de ellos. Las variables ecocardiográficas empleadas para la evaluación de las funciones sistólica y diastólica del ventrículo izquierdo no mostraron diferencias de interés entre los dos grupos, lo cual demuestra su homogeneidad en ese sentido. Resalta el hecho de que los pacientes seleccionados en la investigación tenían una FEVI normal (Tabla 1). 
Tabla 1. Variables ecocardiográficas de acuerdo con la enfermedad coronaria

\begin{tabular}{|l|c|c|c|}
\hline $\begin{array}{l}\text { Variables } \\
\text { ecocardiográficas }\end{array}$ & $\begin{array}{c}\text { ECNS (n= 11) } \\
\text { Media } \pm \text { SD }\end{array}$ & $\begin{array}{c}\text { ECS (n= 25) } \\
\text { Media } \pm \text { SD }\end{array}$ & $p$ \\
\hline Tabique diástole & $10.8 \pm 2.5$ & $11.3 \pm 1.8$ & 0.415 \\
\hline Tabique sístole & $15.6 \pm 2.9$ & $15.1 \pm 2.4$ & 0.961 \\
\hline P. posterior diástole & $10.3 \pm 1.3$ & $11.3 \pm 2.4$ & 0.075 \\
\hline P. posterior sístole & $15.7 \pm 2.2$ & $16.9 \pm 3.6$ & 0.248 \\
\hline VI diástole & $45.8 \pm 4.9$ & $45.8 \pm 6.4$ & 0.998 \\
\hline VI sístole & $29.3 \pm 5.2$ & $30.5 \pm 7.9$ & 0.537 \\
\hline VolTD & $88.5 \pm 27.9$ & $99.9 \pm 36.0$ & 0.552 \\
\hline VolTS & $37.0 \pm 14.4$ & $40.1 \pm 20.1$ & 0.773 \\
\hline FEVI (Simpson) & $55.5 \pm 7.5$ & $61.6 \pm 7.5$ & 0.183 \\
\hline Fac & $34.9 \pm 7.4$ & $34.8 \pm 8.2$ & 0.878 \\
\hline VolTD & $83.1 \pm 32.8$ & $93.9 \pm 23.9$ & 0.458 \\
\hline VolTS & $30.8 \pm 11.8$ & $36.4 \pm 11.4$ & 0.125 \\
\hline FEVI & $62.6 \pm 8.3$ & $60.7 \pm 6.5$ & 0.371 \\
\hline Onda E & $72.7 \pm 13.5$ & $82.1 \pm 19.1$ & 0.127 \\
\hline Onda A & $72.2 \pm 14.9$ & $80.6 \pm 20.3$ & 0.157 \\
\hline Relación E/A & $1.0 \pm 0.3$ & $1.1 \pm 0.5$ & 0.594 \\
\hline VPM & $49.7 \pm 24.1$ & $47.0 \pm 12.0$ & 0.794 \\
\hline E/e' & $10.2 \pm 3.6$ & $9.6 \pm 4.4$ & 0.468 \\
\hline ' & $6.7 \pm 1.5$ & $7.2 \pm 2.4$ & 0.265 \\
\hline Po & $8.1 \pm 3.7$ & $9.7 \pm 4.0$ & 0.650 \\
\hline
\end{tabular}

P. posterior diástole: pared posterior en diástole; P. posterior sístole: pared posterior en sístole; VolTD: volumen telediastólico; VolTS: volumen telesistólico; Fac: fracción de acortamiento; VPM: velocidad de propagación mitral.

En la tabla 2 y la figura 1 se muestran las medias y desviación estándar de los valores de la deformación longitudinal global (DLG) con base en la presencia de enfermedad coronaria significativa o no. La DLG fue menor entre los pacientes con ECS que entre los que tenían ECNS (-20.0 \pm 3.2 vs. $-22.1 \pm 3.6 ; p=0.035)$. Igual comportamiento tuvo esta variable cuando se analizó por vistas ${ }^{2} \mathrm{C}(-19.7 \pm 4.5$ vs. $-23.0 \pm 4.1$; $p=0.008) ; 4 C(-20.7 \pm 3.7$ vs. $-21.6 \pm 3.6, p=0.381)$; у $3 \mathrm{C}(-19.4 \pm 3.1$ vs. $-22.2 \pm 4.8, p=0.016)]$. Aunque existen diferencias entre los dos grupos, hay valores que se superponen entre sí, lo cual hace difícil encontrar un valor de corte para diferenciarlos.

En la figura 2 se muestra la curva ROC para la predicción de ECS mediante DLG. El área bajo la curva es baja, lo cual no permite establecer valores para definir

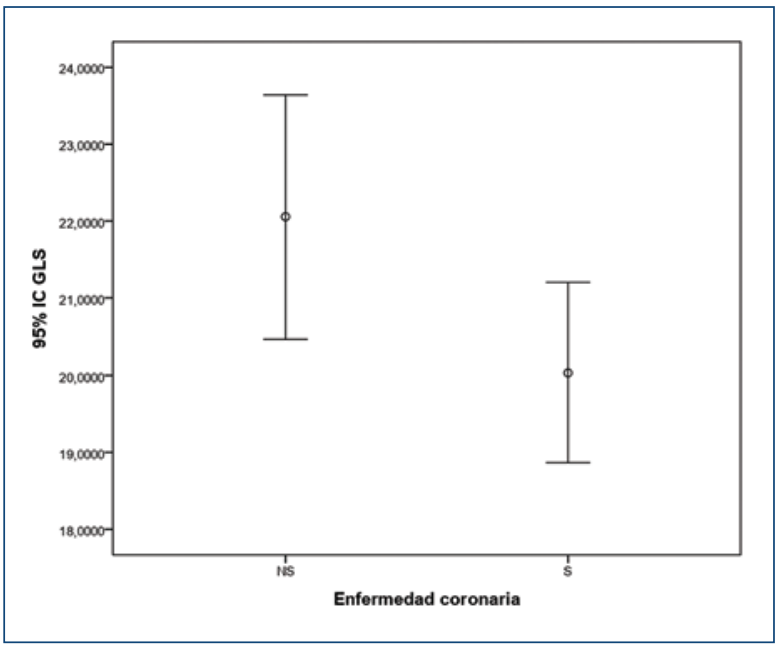

Figura 1. Deformación miocárdica longitudinal global de acuerdo con la enfermedad coronaria.

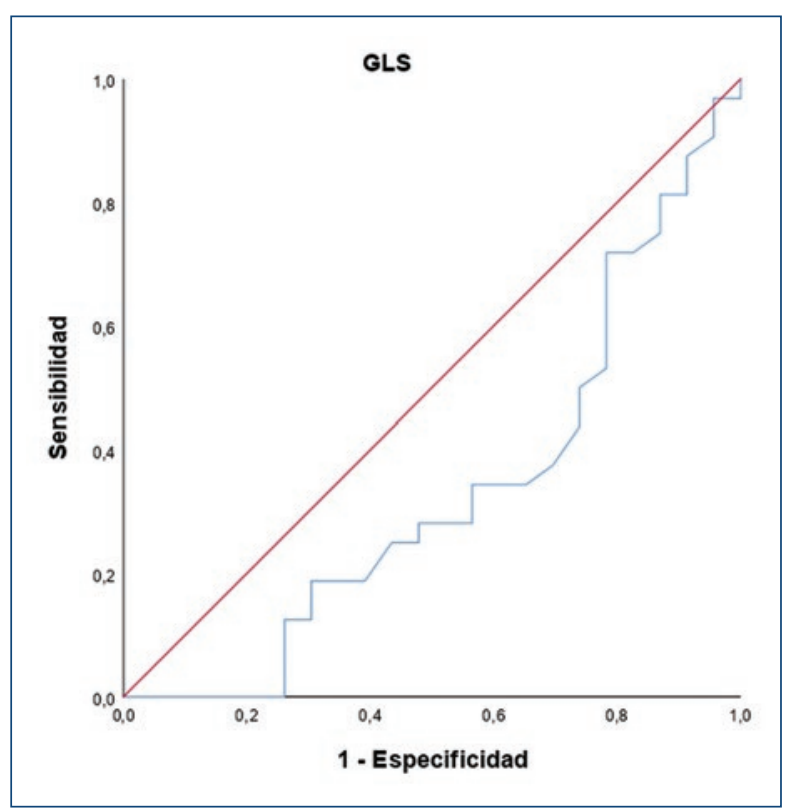

Figura 2. Curvas ROC para la predicción de ECS.

a los pacientes con grados de sensibilidad y especificidad que permitan emplear la técnica con ese fin.

Al analizar la deformación miocárdica global longitudinal de acuerdo con el número de vasos con lesiones angiográficamente significativas se advirtió que no hubo diferencias entre los grupos en ninguno de los casos (Fig. 3).

La tabla 3 muestra las deformaciones promedios de los segmentos cardíacos relacionados con los posibles territorios de irrigación de las arterias 


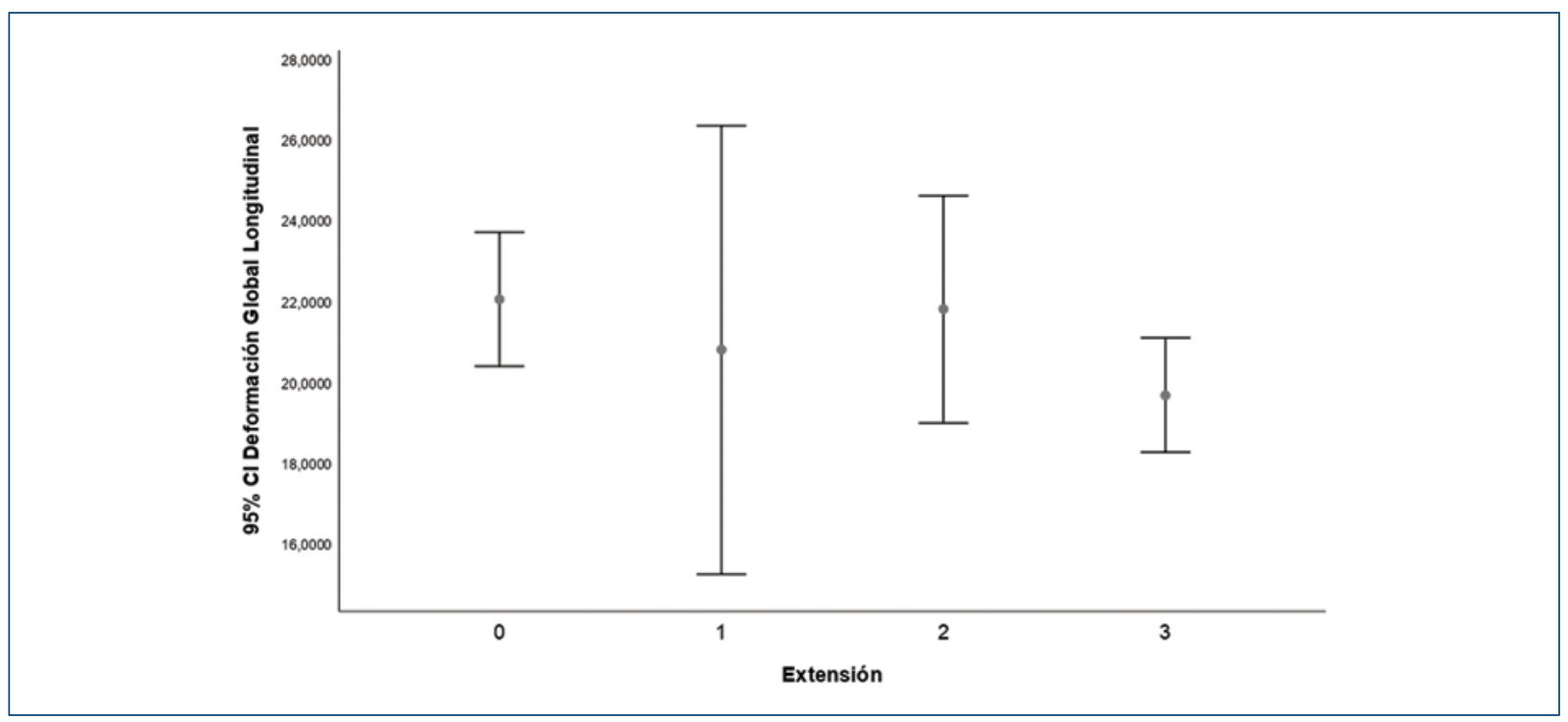

Figura 3. Deformación miocárdica longitudinal global con base en la extensión de la enfermedad coronaria.

Tabla 2. Relación de las deformaciones longitudinales globales y segmentarias de acuerdo con la enfermedad coronaria

\begin{tabular}{|l|l|l|c|}
\hline $\begin{array}{l}\text { Variables speckle } \\
\text { tracking } \\
\text { longitudinal }\end{array}$ & $\begin{array}{c}\text { ECNS }(\mathrm{n}=23) \\
\text { Media } \pm \text { SD }\end{array}$ & $\begin{array}{l}\text { ECS }(\mathrm{n}=32) \\
\text { Media } \pm \text { SD }\end{array}$ & $\boldsymbol{p}$ \\
\hline DLG & $-22.1 \pm 3.6$ & $-20.0 \pm 3.2$ & 0.035 \\
\hline DLG 2C & $-23.0 \pm 4.1$ & $-19.7 \pm 4.5$ & 0.008 \\
\hline DLG 4C & $-21.6 \pm 3.6$ & $-20.7 \pm 3.7$ & 0.381 \\
\hline DLG 3C & $-22.2 \pm 4.8$ & $-19.4 \pm 3.1$ & 0.016 \\
\hline
\end{tabular}

DLG: global longitudinal strain; DLG 2C: global longitudinal strain 2 cámaras; DLG 4C: global longitudinal strain 4 cámaras; DLG 3C: global longitudinal strain 3 cámaras.

epicárdicas principales: descendente anterior, circunfleja y coronaria derecha, según un patrón anatómico previamente establecido y en apariencia uniforme. Los segmentos relacionados con arterias significativamente enfermas tuvieron valores promedios de deformaciones longitudinales menores que los que no tenían arterias con obstrucción significativa, si bien no mostraron diferencias estadísticamente significativas.

\section{Discusión}

En este estudio predominó el sexo masculino, lo cual concuerda con el hecho de que la mayor proporción de los pacientes tenía edades de 50 a 69 años, lapso
Tabla 3. Deformaciones promedio de los segmentos miocárdicos en relación con el área de irrigación de la arteria coronaria y la enfermedad coronaria

\begin{tabular}{|c|c|c|c|}
\hline \multirow[t]{2}{*}{$\begin{array}{l}\text { Variables speckle } \\
\text { tracking }\end{array}$} & $\begin{array}{c}\text { ENS } \\
\text { Media } \pm \text { SD }\end{array}$ & $\begin{array}{c}\text { ES } \\
\text { Media } \pm \text { SD }\end{array}$ & \multirow[t]{2}{*}{$p$} \\
\hline & $n=25$ & $n=28$ & \\
\hline \multirow[t]{2}{*}{$\begin{array}{l}\text { Descendente anterio } \\
\text { Deformación } \\
\text { longitudinal }\end{array}$} & $-21.6 \pm 3.5$ & $-19.9 \pm 3.4$ & 0.093 \\
\hline & $n=31$ & $\mathrm{n}=22$ & \\
\hline \multirow[t]{2}{*}{$\begin{array}{l}\text { Circunfleja } \\
\text { Deformación } \\
\text { longitudinal }\end{array}$} & $-20.7 \pm 3.4$ & $-19.1 \pm 3.3$ & 0.113 \\
\hline & $n=32$ & $n=21$ & \\
\hline $\begin{array}{l}\text { Coronaria derecha } \\
\text { Deformación } \\
\text { longitudinal }\end{array}$ & $-19.6 \pm 3.4$ & $-17.9 \pm 2.7$ & 0.092 \\
\hline
\end{tabular}

ENS: enfermedad no significativa; ES: enfermedad significativa.

en el que las enfermedades cardiovasculares son más frecuentes en el hombre, dado que la mujer está más protegida por factores hormonales conocidos en esta etapa de la vida ${ }^{18,19}$

Este resultado coincide con los obtenidos por grupos del Instituto de Cardiología y Cirugía Cardiovascular (ICCCV), del Hospital Ameijeiras, y del Cardiocentro Ernesto Guevara, de Villa Clara, tanto en relación con el sexo como con las edades de los pacientes ${ }^{20-22}$. 
Se identificó entre los sujetos con ECS una alta frecuencia de los factores de riesgo. La HTA fue la más identificada. Las cifras de presión arterial se correlacionan con la incidencia de enfermedad coronaria y enfermedad cerebrovascular ${ }^{23,24}$.

Según los resultados de la III Encuesta Nacional sobre Factores de Riesgo (III ENFR), la prevalencia global de HTA en Cuba es de $30.9 \%$ en la población $\geq 15$ años, con un incremento a partir de los 55 años. Estos resultados son similares a los publicados en la mayor parte de los países europeos ${ }^{1,25}$.

El tabaquismo es un importante factor de riesgo relacionado con el daño endotelial y la formación de placas en las arterias coronarias. En realidad, un 29\% de las muertes debidas a cardiopatía isquémica tiene como principal causante al tabaco $25-27$.

El hecho de que las tres cuartas partes de los pacientes tengan el diagnóstico clínico de angina crónica estable resulta de gran importancia en el momento de interpretar los resultados, ya que la muestra de estudio la integraron los pacientes sometidos a coronariografía en el laboratorio de hemodinámica del CIMEQ, sitio donde los pacientes agudos son menos frecuentes, por lo que su representación dentro de la muestra fue más baja.

Las tres cuartas partes de los investigados con ECS tenían enfermedad de tres vasos, lo cual se relaciona con una mayor proporción de miocardio isquémico y por tanto justificaría la realización de la coronariografía en la estratificación del riesgo del paciente ${ }^{4}$.

Se ha planteado que episodios repetidos de isquemia miocárdica transitoria generan pequeños grados de aturdimiento miocárdico que, si bien no son suficientes para producir alteraciones visuales en la contractilidad segmentaria, sí pueden modificar la deformación longitudinal. Esto justifica los numerosos estudios realizados que comparan la deformación miocárdica en reposo entre pacientes con ECS y sujetos con ECNS ${ }^{10,13,28,29}$.

Amundsen, et al. ${ }^{30}$, validaron la aplicación del ST bidimensional para su uso clínico al proporcionar medidas precisas, lo que lo convirtió en una potencial herramienta clínica a la cabecera del paciente para cuantificar la función miocárdica regional.

En la investigación realizada se observó que los valores de la deformación miocárdica global longitudinal fueron menores en los pacientes con ECS respecto de aquéllos con enfermedad no significativa, lo cual coincide con varios estudios publicados ${ }^{10,13,28,29,31}$.

Biering-Sorensen, et al. ${ }^{32}$ hallaron que, en pacientes con sospecha de angina de pecho estable, la DLG fue significativamente menor en pacientes con la EAC en comparación con los que no la tenían.

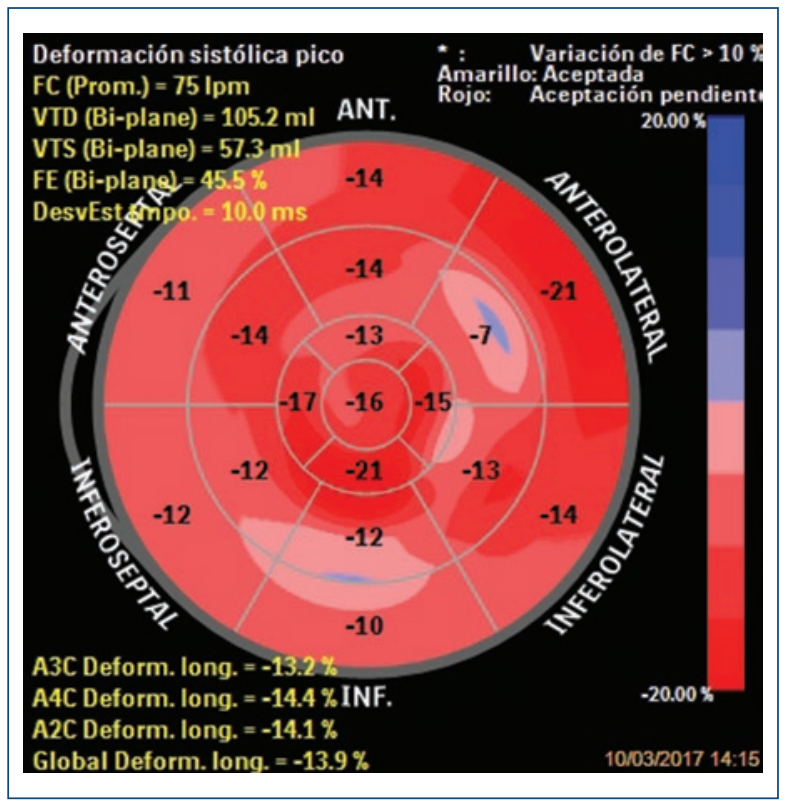

Figura 4. Mapa polar de la deformación miocárdica longitudinal global en un paciente con diagnóstico clínico de angina de empeoramiento progresivo.

Por otra parte, Choi, et al. ${ }^{33}$ propusieron su utilidad para predecir enfermedad de tronco o de tres vasos, en la cual, según sea el área bajo la curva, -17.9\% podría emplearse para diferenciar la EAC grave con sensibilidad y especificidad de $79 \%$.

Aunque en el estudio la diferencia entre los grupos es significativa, es importante señalar que en el grupo de pacientes con ECNS existen enfermos con posible daño microvascular, flujos coronarios lentos o lesiones en una o más arterias coronarias que no sobrepasan el $50 \%$ de la luz del vaso, pero que en sí son pacientes enfermos, por lo que los valores de la deformación miocárdica pueden estar disminuidos (figuras 4 y 5) y puede que se superpongan con los que tienen lesiones coronarias significativas. La presencia de factores de riesgo provoca disrupción en la matriz intersticial miocárdica debido a la isquemia microvascular, la fibrosis y la sustitución colágena, lo cual puede llevar a cambios microscópicos en la fibra miocárdica que generan disfunción miocárdica subclínica, potencialmente detectable a través del estudio de la deformación miocárdica ${ }^{34}$.

Con base en lo anterior puede explicarse que, al analizar la curva COR, el área bajo ella no justifica la aplicación de la herramienta para discernir entre el paciente con arterias coronarias significativamente enfermas del paciente en que las coronarias pueden estar 


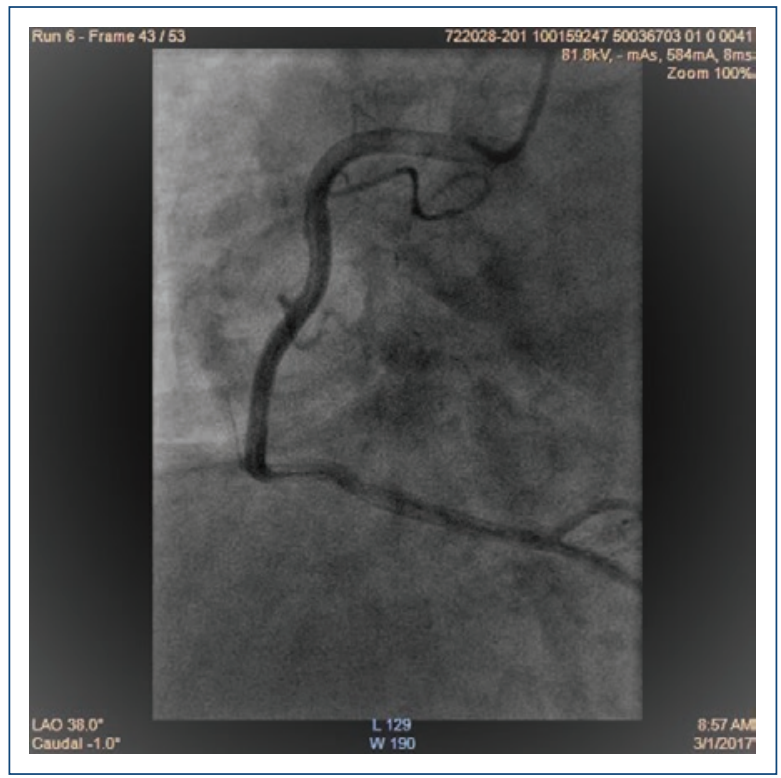

Figura 5. Imagen de cateterismo cardíaco en arteria coronaria derecha con flujo coronario lento.

normales 0 apenas obstruidas, dado que los valores se superponen entre sí.

Respecto de las deformaciones miocárdicas, y en relación con las arterias epicárdicas y sus posibles zonas de irrigación, aunque fueron menores en los segmentos relacionados con arterias con obstrucción significativa, sus diferencias no fueron relevantes en términos estadísticos. La anatomía coronaria es variable, por lo que valorar la función territorial de acuerdo con un área vascular específica puede no reflejar la verdadera distribución arterial coronaria. Norum, et al. ${ }^{35}$ publicaron resultados similares.

Aunque la base de evidencia para su uso en ecocardiografía clínica habitual es mucho menor que la de la $\mathrm{FEVI}$, se ha demostrado en varios estudios que las medidas de DLG son sólidas y reproducibles. La DLG es un predictor más potente de todas las causas de mortalidad comparada con la FEVI, de acuerdo con 16 estudios con un total de 5,721 pacientes ${ }^{36}$. No obstante, la FEVI es todavía el principal parámetro para la evaluación de la función cardíaca. La DLG es en particular importante en pacientes con FEVI normal o mínimamente disminuida, al ser más sensible para la detección temprana de la enfermedad miocárdica ${ }^{37,38}$.

Sin embargo, aún no son posibles su extensión y aplicación, en especial debido a que no hay acuerdo actual acerca de su utilidad debido a la variabilidad en el software y los proveedores, además de la necesidad de unificar criterios en relación con su realización por parte de los vendedores de los equipos capaces de realizarla ${ }^{10}$.

Por último, el mayor beneficio del empleo del ST 2D es que hace a la ecocardiografía más objetiva y cuantitativa, lo cual la convierte en una estudio cardíaco más completo y exacto. Muchos estudios complementarios pueden ser costosos. A pesar de las limitaciones tecnológicas actuales, es probable que la DLG se convierta en una herramienta clínica estándar en el futuro próximo, en la medida en que los expertos en imágenes adquieran confianza en su medición y los clínicos se familiaricen más con su valor. Para ser efectiva en términos del costo, la ecocardiografía debe ser un estudio definitivo ${ }^{37}$. El uso de ST 2D es un gran paso en esa dirección.

En la investigación realizada en pacientes con cardiopatía isquémica predominaron los hombres en la sexta década de la vida. Los factores de riesgo más frecuentes fueron la HTA y el tabaquismo, este último presente en más de la mitad de los pacientes con ECS. La mayor proporción de pacientes tenía el diagnóstico clínico de angina crónica estable. Los valores de la ecocardiografía bidimensional evidenciaron datos similares en pacientes con ECNS y ECS. Los valores de DLG fueron menores en los sujetos con ECS, así como los valores segmentarios en relación con las arterias afectadas. No hubo diferencias en la DLG según el número de vasos significativamente enfermos. A pesar de lo anterior, los modestos resultados no justifican el empleo del ST 2D para diferenciar la ECS.

\section{Financiamiento}

Esta investigación no ha recibido ninguna beca específica de agencias de los sectores público, comercial, o sin ánimos de lucro.

\section{Conflicto de intereses}

Los autores declaran no tener ningún conflicto de intereses.

\section{Responsabilidades éticas}

Protección de personas y animales. Los autores declaran que para esta investigación no se han realizado experimentos en seres humanos ni en animales.

Confidencialidad de los datos. Los autores declaran que han seguido los protocolos de su centro de trabajo sobre la publicación de datos de pacientes. 
Derecho a la privacidad y consentimiento informado. Los autores han obtenido el consentimiento informado de los pacientes o sujetos referidos en el artículo. Este documento obra en poder del autor de correspondencia.

\section{Bibliografía}

1. American Heart Association. Resumen de estadísticas de 2017. Enfermedad del corazón y ataque cerebral [Internet]. Comité de Estadísticas y del Subcomité de Estadísticas de Ataque [citado 17 enero 2017]. Disponible en: https://www.heart.org/idc/groups/ahamahpublic/@wcm/@ sop/@smd/documents/downloadable/ucm_491392.pdf

2. Dirección de Registros Médicos y Estadísticas de Salud. Anuario Estadístico de Salud 2016 [Internet]. La Habana: MINSAP; 2017 [citado 5 jul 2017]:206-5 p. Disponible en: http://files.sld.cu/dne/files/2017/05/ Anuario_Estad\%C3\%ADstico_de_Salud_e_2016_edici\%C3\%B3n_2017.pdf

3. Trainini JC, Elencwajg, López Cabanillas N, Herrero J, Lago N, Lowenstein JA, et al. Fundamentos de la nueva mecánica cardíaca. La bomba de succión. 1ra. Buenos Aires: Lumen; 2015:112p.

4. Montalescot G, Sechtem U, Achenbach S, Andreotti F, Arden C, Budaj A, et al. Guía de Práctica Clínica de la ESC 2013 sobre diagnóstico y tratamiento de la cardiopatía isquémica estable. Rev Esp Cardiol. [Internet] 2014 Feb [citado 5 Jul 2017];67(2): 135.e1-8.Disponible n: http://www. sacardiologia.com/docs/cardioteca/cardioteca1/7.pdf.

5. Marwick TH. Ejection fraction pros and cons [abstract]. J Am Coll Cardiol. [Internet] 2018 Nov [cited 2018 Nov 30];72(19): [about 2 p.]. Available from: http://www.onlinejacc.org/content/72/19/2360?download = true.

6. Cossío PR. Fundamentos de la nueva mecánica cardíaca. La bomba de succión. Crítica bibliográfica. Rev Argent Cardiol. [Internet] 2016 Feb [citado 23 Ene 2017];84(1):1. Disponible en: http://www.scielo.org.ar/pdf/ rac/v84n1/v84n1a16.pdf

7. Poveda F, Gil D, Martí E, Andaluz A, Ballester M, Carreras F. Estudio tractográfico de la anatomía helicoidal del miocardio ventricular mediante resonancia magnética por tensor de difusión. Rev Esp Cardiol. [Internet] 2013 Oct [citado 10 Nov 2018];66(10):782-90. Disponible en: http:// www.revespcardiol.org/es/pdf/90227055/S300/.

8. Kocabay G, Muraru D, Peluso D, Cucchini U, Sorina M, Padayattil-José S, et al. Mecánica ventricular izquierda normal mediante ecocardiografía speckle tracking bidimensional. Valores de referencia para adultos sanos [resumen]. Rev Esp Cardiol. [Internet] 2014 Ago [citado 22 Nov 2018];67(8): [aprox. 3 p.]. Disponible en: https://www.sciencedirect.com/science/article/pii/S030089321400089X?via\%3Dihub.

9. Mada RO, Duchenne J, Voigt JV. Tissue Doppler, strain and strain rate in ischem ic heart disease "How I do it". Cardiovasc Ultrasound [Internet] 2014 Sep [cited 2018 Nov 22]: [about 42 p.]. Available from: https://www. ncbi.nlm.nih.gov/pmc/articles/PMC4177059

10. Voigt JU, Pedrizzetti G, Lysyansky P, Marwick T, Houle H, Baumann R, et al. Definitions for a common standard for 2D speckle tracking echocardiography: Consensus Document of the EACVI/ASE/Industry Task Force to Standardize Deformation Imaging. Eur Heart J Cardiovasc Imaging [Internet]. 2015 Jan [cited 2018 Jan 15];16(1):1-11. Available from: https://academic.oup.com/ehjcimaging/article-pdf/16/1/1/7140604/ jeu184.pdf

11. Takeuchi M, Nishikage T, Nakai H, Kokumai M, Otani S, Lang RM. The assessment of left ventricular twist in anterior wall myocardial infarction using two dimensional speckle tracking imaging [abstract]. J Am Soc Echocardiogr. [Internet]. 2007 Jan [cited 2018 Jan 15];20(1): [about 2 p.] Available from: https://www.sciencedirect.com/science/article/pii/ S0894731706006638.

12. Billehaug I, Ruddox V, Edvardsen T, Otterstad JE. Diagnostic accuracy of left ventricular longitudinal function by speckle tracking echocardiography to predict significant coronary artery stenosis. A systematic review. BMC Med Imaging [Internet]. 2015 Jul [cited 2018 Jan 13]: [about 30 p.]. Available from: https://bmcmedimaging.biomedcentral.com/articles/10.1186/s12880-015-0067-y.

13. Damman $P$, van Geloven $N$, Walentin L, Lagerqvist B, Fox CA, Clayton $T$, et al. Timing of angiography with a routine invasive strategy and longterm outcomes in non-ST segment evaluation acute coronary syndrome: a collaborative analysis of individual patient data from the FRISC II (Framingham Fast Revascularization During Instability in Coronary Artery Disease), ICTUS (Invasive versus conservative Treatment in Unstable Coronary Syndromes), and RITA-3 (Intervention vs Conservative treatment Strategy in Patients With Unstable Angina or Non-ST elevation Myocardial Infarction) Trial. J Am Coll Cardiol Intv. [Internet] 2012 Feb [cited 2018 Jan 21];5(2): [about 22 p.]. Available from: http://interventions. onlinejacc.org/content/5/2/191.
14. Hamm CW, Bassand JP, Agewall S, Bax J, Boersma E, Bueno H, et al. ESC Guidelines for the management of acute coronary syndromes in patients presenting without persistent ST-segment elevation: the Task Force for the management of acute coronary syndromen (ACS) in patients presenting without persistent ST-segment elevation of the European Society of Cardiology [abstract]. Eur Heart J. [Internet] $2011 \mathrm{Mar}$ [cited 2018 Jan 15];13(3):[about 1 p.]. Available from: https://www.ncbi. nlm.nih.gov/pubmed/22395108.

15. Gastaldello A, Merlo P, Amor M, Alasia D, Galello M, Rousse M, et al. Longitudinal strain at rest does not predict stress echo results. Rev Argent Cardiol. [Internet] 2016 Aug [cited 2017 Sep 25];84(4):330-5. Available from: https://www.sac.org.ar/wp-content/uploads/2016/09/ v84n4a11-en.pdf.

16. Caniggia C, Amor M, Lowenstein Haber D, Alasia D, Galello M, Darú V, et al. Factibilidad y aportes del análisis de la deformación longitudinal 2D global y regional durante el eco estrés con ejercicio. Rev Argent Cardiol. [Internet] $2014 \mathrm{Abr}$ [citado 25 May 2017];82(2):110-7. Disponible en: https://www. redalyc.org/pdf/3053/305331222010.pdf.

17. Lang RM, Badano LP, Mor-Avi V, Afilalo J, Armstrong A, Ernande L, et al. Recommendations for cardiac chamber quantification by echocardiography in adults: an update from the American Society of Echocardiography and the European Association of Cardiovascular Imaging. J Am Soc Echocardiogr. [Internet] 2015 Oct [cited 2017 Jan 15];82(4): [1-39.]. Available from: https://orbi.uliege.be/bitstream/2268/221545/1/jase\%20lang.pdf

18. Redberg R, Benjamin E, Bittner V, Braun L, Goff D, Havas S, et al. ACCF/ AHA 2009 performance measures for primary prevention of cardiovascular disease in adults. JACC Cardiovasc Imaging [Internet] 2009 Sep [cited 2017 Sep 11];54(14): 1364-405. Available from: http://www.onlinejacc. org/content/accj/54/14/1364.full.pdf?download = true.

19. Armas N, Dueñas A, de la Noval R, Ortega Y, Acosta M, Morales A. Cardiopatía Isquémica en Cuba. Una puesta al día. 2015. Rev Cuban Cardiol. [Internet] 2015 [citado 26 Feb 2017];21(3):133-8. Disponible en: http://www. revcardiologia.sld.cu/index.php/revcardiologia/article/view/597/pdf_15

20. Villar A, Ocaña H, Pedroso J, Cruz R, Cosimo M, Peña E, et al. Primeros resultados comparativos de dos vías de abordajes de cirugía de revascularización coronaria mínimamente invasiva. Rev Cuba Cir. [Internet] 2010 Oct-Dic [citado 23 Ene 2017];49(4): [aprox. 23 p.]. Disponible en: http://scielo.sld.cu/scielo.php?script = sci_arttext\&pid = S003474932010000400003.

21. Santos A, Pérez H, Valero A, Lage L, Borges R. Factores preoperatorios relacionados con las complicaciones cardiovasculares de la cirugía de revascularización coronaria. Gac Méd Espirituana [Internet] 2010 [citado 6 Abr 2017];12(3): [aprox. 11 p.]. Disponible en: http://bvs.sld.cu/revistas/ gme/pub/vol.12.\%283\%29_06/p6.html.

22. Echemendía ED, Villar A, Nafeh M, Pedroso J, Martí D, Hidalgo T. Comparación de los resultados de la revascularización miocárdica con corazón latiente, con circulación extracorpórea y sin ella. Rev Cuba Cir. [Internet] 2011 Ene-Mar [citado 6 Abr 2017];50(1):[aprox. 29 p.]. Disponible en: http:// scielo.sld.cu/scielo.php?script=sci arttext\&pid = S0034-74932011000100003

23. Organización Mundial de la Salud OMS [Internet]. Suiza; c2018. Enfermedades cardiovasculares; citado 16 Jun 2017]; [aprox. 18 pantallas]. Disponible en: https://www.who.int/es/news-room/fact-sheets/detail/cardiovascular-diseases-(cvds).

24. Paula Piñeira BM, Díaz Valdez YN, Hernández Puentes Y, Salinas Olivares M, Almeida Carralero G, Gutiérrez Alba NE, et al. Impacto de la hipertensión arterial en el proceso aterosclerótico de las arterias coronarias: patomorfometría. Rev Cuban Invest Bioméd. [Internet] 2009 Jul-Sep [citado 20 Mar 2016];28(3):37-43. Disponible en: http://scieloprueba.sld. cu/pdf/ibi/v28n3/ibi04309.pdf.

25. Bonet M, Varona P, La Rosa MC, García RG, Suárez R, Arcia N, et al. III Encuesta nacional de factores de riesgo y actividades preventivas de enfermedades no trasmisibles [Internet]. Cuba 2010-2011. La Habana: Ciencias Médicas; 2014. [Citado 23 Mar 2017]. 329 p. Disponible en: https://www.researchgate.net/publication/325370475.

26. Mahmood SS, Levy D, Vasan RS, Wang TJ. The Framingham Heart Study and the epidemiology of cardiovascular disease: a historical perspective. Lancet [Internet] 2014 Mar [cited 2017 Jul 20];383(9921):9991008. Available from: https://www.ncbi.nlm.nih.gov/pmc/articles/ PMC4159698/pdf/nihms588573.pdf.

27. O'Donnell CJ, Elosua R. Factores de riesgo cardiovascular. Perspectivas derivadas del Framingham Heart Study. Rev Esp Cardiol [Internet]. 2008 Mar [citado 25 Jul 2017];():199-310. Disponible en: https://www. sciencedirect.com/science/article/pii/S0300893208733888.

28. Montgomery DE, Puthumana JJ, Fox JM, Ogunyakin KO. Global longitudinal strain aids the detection of non-obstructive coronary artery disease in the resting echocardiogram. Eur Heart $\mathrm{J}$ Cardiovasc Imaging [Internet] 2012 Jul [cited 2018 Jan 15];13(7): [about 44 p.]. Available from: https://academic.oup.com/ehjcimaging/article/13/7/579/2397234

29. Smedsrud MK, Sarvari S, Haugaa KH, Gjesdal O, Orn S, Aaberge L, et al. Duration of myocardial early systolic lengthening predicts the presence of significant coronary artery disease. J Am Coll Cardiol. [Internet] 2012 Sep [cited 2018 Jan 15];60(12): 1086-93. Available from: http:// www.onlinejacc.org/content/accj/60/12/1086.full.pdf?download = true. 
Arch Cardiol Mex. 2020;90(3)

30. Amundsen BH, Helle-Valle T, Edvardsen T, Torp H, Crosby J, Lyseggen E, et al. Noninvasive myocardial strain measurement by speckle tracking echocardiography: Validation against sonomicrometry and tagged magnetic resonance imaging. J Am Coll Cardiol. [Internet] $2006 \mathrm{Feb}$ [cited 2017 Jan 23];47(4):789-93. Available from: https://www.sciencedirect. com/science/article/pii/S0735109705027506/pdfft?md5 = 3758a9cd0cb849b3899667fad6fe11ba\&pid = 1-s2.0-S0735109705027506-main.pdf

31. Shimoni S, Gendelmann G, Ayzenberg O, Smirin N, Lysyansky P, Edri O, et al. Differential effects of coronary artery stenosis of myocardial function: the value of myocardial strain analysis for the detection of coronary artery disease. J Am Soc Echocardiogr. [Internet] 2011 Jul [cited 2018 Jan 15];24(7):748-57. Available from: https://www.sciencedirect. com/science/article-pdf/pii/S0894731711001908.pdf.

32. Biering-Sørensen $T$, Hoffman S, Mogelvang R, Zeeberg Iversen A Galatius S, Fritz-Hansen T, et al. Myocardial strain analysis by 2-Dimensional speckle tracking echocardiography improves diagnostics of coronary artery stenosis instable angina pectoris. Circ Cardiovasc Imaging [Internet] 2014 Jan [cited 2018 Jan 15];7(1):58-65. Available from: https://www. ahajournals.org/doi/pdf/10.1161/CIRCIMAGING.113.000989.

33. Choi J, Cho SW, Bin Y, Jin S, Gun B, Lee S, et al. Longitudinal 2D strain at rest predicts the presence of left main and three vessels coronary artery disease in patients without regional wall motion abnormality. Eur J Echocardiog. [Internet] 2009 Jul [cited 2017 Jan 23]; 10(5):695-701. Available from: https://academic.oup.com/ehjcimaging/article-pdf/10/5/ 695/7117757/jep041.pdf.
34. Gaibazzi N, Pigazzani F, Reverberi C, Porter TR. Rest global longitudinal $2 \mathrm{D}$ strain to detect coronary artery disease in patients undergoing stress echocardiography: a comparison with wall-motion and coronary flow reserve responses. Echo Res Pract. [Internet] 2014 Dec [cited 2017 Jan 23];1(2):61-70. Available from: https://www.ncbi.nlm.nih.gov/pmc/articles/ PMC4676453/pdf/echo-01-61.pdf.

35. Norum I, Ruddox V, Edvardsen T, Otterstad JE. Diagnostic accuracy of left ventricular longitudinal function by speckle tracking echocardiography o predict significant coronary artery stenosis. a systematic review. BMC Med Imaging [Internet] 2015 Jul [cited 2018 Jan 15];15(25): [about 42 p.]. Available from: https://www.ncbi.nlm.nih.gov/pmc/articles/PMC4513709/.

36. Feigenbaum H, Mastouri R, Sawada S. Practical approach to using strain echocardiography to evaluate the left ventricle. Circ J. [Internet] 2012 [cited 2018 Ene 21]; 76:1550-5. Available from: https://www.jstage.jst. go.jp/article/circj/76/7/76 CJ-12-0665/_pdf.

37. Otto CM. Heartbeat. Highlights from this issue Heart [Internet]. Tomo 100 Vol. 18. London; 2014 Sep [cited 2017 Jan 23]. [about 1397 p.]. Available from: https://search.proquest.com/openview/31d51395b17a6897076b5d87dbebceca/1 ?pq-origsite $=$ gscholar\&cbl $=2041068$

38. Stokke T, Hasselberg N, Smedsrud M, Sarvari S, Haugaa K, Smiseth O, et al. Geometry as a confounder when assessing ventricular systolic function comparison between ejection fraction and strain. J Am Coll Cardiol. [Internet] 2017 Aug [cited 2018 Jan 23];70(8):942-54. Available from: https://www.sciencedirect.com/science/article/pii/S07351097 17379391/pdfft?md5 = 2afca1bd83acbb636a0f6acd5752243b\&pid $=$ 1-s2.0-S0735109717379391-main.pdf 